\title{
NUESTRA EXPERIENCIA EN EL EMPLEO DE DIFERENTES MATERIALES INYECTABLES EN EL TRATAMIENTO ENDOSCÓPICO DEL REFLUJO VESICOURETERAL
}

\author{
Daniel Cabezalí Barbancho, Andrés Gómez Fraile, Francisco López Vázquez, María López Díaz y \\ Adolfo Aransay Bramtot.
}

Sección de Urología. Servicio de Cirugía Pediátrica. Sección de Medicina Nuclear. Servicio de Radiología Hospital Universitario 12 de Octubre. Madrid. España.

\begin{abstract}
Resumen.- OBJETIVO: El Reflujo Vésico Ureteral (RVU) es la anomalía urológica más común en la infancia, afectando al $1 \%$ de la población pediátrica. El tratamiento endoscópico del RVU está aceptado como la primera opción terapéutica del mismo y desde su implantación se han utilizado diferentes materiales inyectables. Exponemos nuestra experiencia en el tratamiento endoscópico del RVU con las distintas sustancias que hemos ido empleando desde que comenzamos a realizar el procedimiento.
\end{abstract}

MÉTODOS: Hemos realizado un estudio retrospectivo descriptivo de los 445 pacientes que han sido tratados endoscópicamente de reflujo vesicoureteral (RVU) en nuestro centro entre los años 1988 y 2004. Hemos tratado un total de 568 uréteres y hemos analizado los resultados en función del material empleado, el grado de reflujo y la patología asociada.
Daniel Cabezalí Barbancho Beasain, 35 - 6으. 28041 Madrid. (España).

dcabezali@yahoo.es
RESULTADOS: De los 569 uréteres con RVU tratados, 457 eran RVU (79\%) simples, 76 (15\%) estaban incluidos en un sistema renal doble, 24 (4\%) se asociaban a vejiga neurógena y 12 (2\%) eran RVU secundarios a cirugía antirreflujo abierta. Utilizamos tres tipos de materiales, predominado el politetrafluoroetileno con 257 uréteres, siguiéndole el dextranómero de ácido hialurónico con 159 y por último el polidimetilsilixano con 153. En los "RVU simples" la tasa de curación global fue del 88\% (38 1 uréteres), con mejora importante del grado de reflujo en el 7\% 151 uréteres), precisando una reimplantación tipo Cohen en el 5,5\% de los casos (25 pacientes). Observamos un menor índice de éxitos y mayor necesidad de más inyecciones en los reflujos grado IV y V. En los "RVU asociados a duplicidad" los resultados empeoran, con menos éxitos y mayor necesidad de procedimientos para su resolución. Curamos 59 uréteres (77\%) sobre 76 uréteres tratados, 13 uréteres (19\%) mejoraron y $4 \%$ precisaron de reimplantación tipo Cohen. En los "RVU asociados a vejiga neurógena" se consiguió curación en 20 uréteres (83\%). Hubo mejoría significativa del reflujo en 2 uréteres (8\%). Fracasamos en dos (8\%), necesitando de reimplantación tipo Cohen para evitar el deterioro progresivo del riñón. En los "RVU de uréteres reimplantados mediante cirugía abierta" curamos 12 uréteres (100\%) sobre 12 tratados. Hubo 8 uréteres simples, solucionando el reflujo en 6 con un único procedimiento, mientras que uno precisó de dos procedimientos. En total el número de uréteres curados ha sido de 496 (87\%) y 51 (9\%) han mejorado. Se han intervenido 22 uréteres (4\%). Con 1 inyección hemos curado un 68\%, con 2 inyecciones un 16,5\% y con 3 el 1\%. Las complicaciones acaecidas en estos 569 procedimientos, fueron 5 (0,8\%): 1 caso de cistitis hemorrágica que cedió espontáneamente en dos días y 4 pielonefritis que se trataron con antibiótico adecuado según antibiograma. No hemos tenido casos de infecciones urinarias bajas de repetición. El tiempo de seguimiento ha variado de 1'5 años a 15 años.

CONCLUSIONES: Parece que tanto el polidemitilsiloxano como el dextranómero de ácido hialurónico son materiales 
buenos y seguros y no tienen el peligro de migración a distancia que tiene el politetrafluoroetileno.

Palabras clave: Tratamiento endoscópico. Materiales inyectables. Reflujo vesicoureteral.

Summary.- OBJETIVES: Vesicoureteral reflux (VUR) is the most common urologic anomaly in childhood, affecting $1 \%$ of the pediatric population. Endoscopic treatment of VUR is accepted as the first therapeutic option and various injectable materials have been used since its implantation. We present our experience in the endoscopic treatment of VUR with various substances which we have been employing since we started performing the procedure.

METHODS: We have performed a retrospective descriptive study including 445 patients that underwent endoscopic treatment for vesicoureteral reflux in our centre between 1988 and 2004. We treated a total of 568 ureters, and we analyze results depending on the material employed, grade of reflux and associated pathology.

RESULTS: Among 569 ureters with vesicoureteral reflux undergoing treatment 457 were single VUR (79\%), 76 (15\%) were part of a double renal system, 24 (4\%) were associated with neurogenic bladder, and 12 (2\%) were secondary VUR after antireflux open surgery. We use three types of materials, with predominance of polytetrafluorethylene in 257 ureters, followed by hyaluronic acid dextranomer in 159, and finally polydimethylsiloxane in 153. In the cases of single VUR global cure rate was $88 \%$ (38 1 ureters), with significant improvement of the grade of reflux in 7\% (51 ureters), and 5.5\% of the cases (25 patients) requiring a Cohen type reimplantation. We observed a lower success rate and greater need of repeated injections in grade IV and V refluxes. In VUR associated with duplication results are worse, with less successes and greater need of procedures for its resolution. We cured 59 ureters (77\%) out of 76 treated, 13 (19\%) improved, and $4 \%$ required Cohen type reimplantation. In cases of VUR associated with neurogenic bladder 20 ureters were cured (83\%). There was significant improvement in two ureters $(8 \%)$; there were two failures (8\%), requiring Cohen type reimplantation to avoid progressive deterioration of the kidney. In the cases of VUR after open surgical reimplantation all 12 ureters treated were cured (100\%). Among 8 single ureters, reflux was solved with 1 procedure in 6 and 7 required 2 procedures. The total number of cured ureters has been 496(87\%), and 51(9\%) have improved. 22 ureters underwent surgery (4\%). $68 \%$ of the cases were cured after 1 injection, $16.5 \%$ after 2 , and $1 \&$ after 3. There have been 5 complications $(0,8 \%)$ : 1 case of hemorrhagic cystitis which resolved spontaneously in two days, and 4 pyelonephritis which received the appropriate antibiotic therapy following antibiogram. We did not have any case of recurrent lower urinary tract infections. Follow-up has range it from 1.5 to 15 years.

CONCLUSIONS: It seems that both polydimethylsiloxane and hyaluronic acid dextranomer are good and safe materials, and do not have the risk of distant migration of polytetrafluorethylene.
Keywords: Endoscopic treatment. Injectable materials. Vesicoureteral reflux.

\section{INTRODUCCIÓN}

El reflujo vésico ureteral (RVU) es la anomalía urológica más común en la infancia, afectando al $1 \%$ de la población pediátrica y al $25-40 \%$ de los que tienen episodios de pielonefritis (1).

Se detecta con mayor frecuencia en niños de raza blanca, con una prevalencia menor del $1 \%$ y una incidencia mayor del $10 \%$. Su causa parece deberse a un desorden anatómico y funcional en la unión urétero-vesical (UUV) (2).

Frecuentemente desaparece durante el crecimiento, aunque su importancia clínica radica en la morbilidad causada por su asociación a infecciones del tracto urinario (ITU) y a la posible nefropatía que puede conducir al deterioro de la función renal entre un $3-25 \%$ de los niños y (3). Desde que en 1893 Pozzi observase el RVU por primera vez en humanos se han publicado numerosos trabajos que han desarrollado su conocimiento (4). A pesar de ello sigue sin existir consenso sobre su manejo.

A partir de 1970 varias publicaciones han comparado prospectivamente el tratamiento médico y quirúrgico del RVU. El estudio de "Birminghan" mostró que más de la mitad de los niños con reflujo de alto grado tratados médicamente, continuaron mostrándolo después de 5 años (5). El estudio de "Toronto" reveló que el 93\% de los pacientes con reflujo grado IV y el $83 \%$ con grado III tenían persistencia del mismo 2 años después de tratamiento médico, y que en un $70 \%$ con grado IV y un $50 \%$ con grado III permanecieron con el mismo reflujo después de 5 años de seguimiento conservador (6). El "Estudio Internacional del Grupo del Reflujo (Sección Europea)" mostró que el 84\% de los niños con RVU grado III y IV tratados médicamente de forma randomizada, continuaron con reflujo después de 5 años, y aquellos con RVU bilateral, el $91 \%$ tenían persistencia del RVU después de 5 años (7). La "Asociación Americana de Urología", reporta una resolución del 9'9\% en los grados IV después de 5 años de tratamiento médico (8). Todos estos estudios también mostraron que el tratamiento conservador conlleva un riesgo continuo de cicatriz renal. El reto consiste en poder contestar a las siguientes preguntas: ¿Cuánto tiempo deben ser seguidos? ¿¿Deben ser manejados con quimioprofilaxis?, ¿̇En qué RVU está indicado el tratamiento quirúrgico?

Existen diversas técnicas para tratar el RVU mediante cirugía abierta. Su principio es aumentar la longitud el uréter intravesical con un sólido soporte detrusoriano, para permitir su compresión por la orina cuando se llena la vejiga. Estas técnicas son efectivas pero están asociadas a morbilidad. Requieren apertura vesical y por tanto obligan a los pacientes a estar ingresados varios días en el hospital. Tiene como complicaciones más frecuentes la recidiva del RVU que se presenta según diferentes series entre el $9-19,3 \%$ y el y la obstrucción que aparecen entre el 0,3\% y el $9 \%(7,8)$. 
Con el objetivo de disminuir la morbilidad asociada a la cirugía, Matouschek en 1981 (9) y en 1984 O’Donell y Puri (10) describieron la corrección endoscópica del RVU y trataron mediante inyección subureteral de pasta politetrafluoroetileno el RVU inducido en cerdos. Posteriormente los mismos autores la utilizaron en niños con éxito, tratando RVU primarios y secundarios, ganando aceptación por múltiples centros y obteniendo tasas de curación del 70 al $90 \%(11,12)$.

En nuestro hospital se realiza este procedimiento desde el año 1988 y al igual que en otros centros se han empleado diversos materiales para la inyección dependiendo de la época. La industria farmaceútica continua buscando el compuesto ideal que cumpla los siguientes requisitos:

1- Capacidad de ser introducido endoscópicamente y conservar su volumen con el tiempo.

2- Que sea biocompatible, no antigénico, no carcinogénico y no migratorio.

Exponemos nuestra experiencia en el tratamiento endoscópico del RVU demostrando su eficacia independientemente del material empleado lo que nos permitiría utilizarlo como arma terapéutica de primer orden para la resolución de esta patología.

\section{MATERIAL Y MÉTODOS}

Hemos realizado un estudio retrospectivo descriptivo de los 445 pacientes que han sido tratados endoscópicamente de reflujo vesicoureteral (RVU) en nuestro centro entre los años 1988 y 2004.

Tratamos 568 uréteres con reflujo primario, asociado a duplicidades, a vejiga neurógena y reflujos no curados tras intervención quirúrgica (Tabla I).

La edad de tratamiento osciló en un rango de 2 meses a 21 años.

En los asociados a vejiga neurógena hubo una selección previa de los pacientes candidatos a esta técnica, ya que debían tener controlado su patología de base: Vejigas con buena capacidad y compliance, comportamiento hipotónico o respuesta buena al tratamiento anticolinérgico confirmado por estudio urodinámico, buen manejo de la enfermedad por el paciente o por la familia.

El diagnóstico de RVU se estableció mediante una cistografía y los controles posteriores mediante ecocistografías. El RVU fue graduado según la clasificación "Internacional del Reflujo" En todos los pacientes se efectuó una ecografía del aparato urinario y una cintigrafía con ácido dimercaptosuccínico (DMSA).

En la ecografía se evaluó el diámetro anteroposterior de la pelvis renal, el grado de dilatación y el grosor de la corteza renal. Con el DMSA valoramos el grado de función renal relativa de cada riñón. Hemos tratado todos aquellos RVU de grados $\|-V$ que se asociaban a una pér- dida de la función renal o a infecciones de repetición, y aquellos que persistían después de los 4 años de edad. Los RVU de grado I solo se trataron cuando se asociaban a un reflujo contralateral que requería tratamiento.

La técnica endoscópica empleada es similar a la original descrita por O'Donnell y Puri (10) Todos recibieron profilaxis antibiótica durante el proceso, generalmente "Cefalosporina de 1 1 generación". Practicamos cistoscopia bajo anestesia general y en posición de litotomía dorsal. La técnica se efectúa a través de un cistoscopio del 9,5 $\mathrm{Fr}$ con canal recto o del 13 o $14 \mathrm{Fr}$ con canal curvo, que permiten el paso del catéter de inyección del 4'5 Fr. Preferimos, si se puede, la lente de 30 grados ya que permite visualizar mejor los meatos ureterales y ver la aguja del catéter nada mas salir del cistoscopio, lo que da mayor precisión a la hora de efectuar la punción.

Una vez localizado el meato refluyente introducimos el catéter situando la punta de la aguja dentro del meato, midiendo su longitud, siendo su final el sitio de la punción mucosa, que suele ser a unos pocos milímetros del orificio ureteral, entre 4 y $6 \mathrm{~mm}$. La aguja se introduce submucosamente en posición de las 6 h, apreciando su recorrido hasta su final en el borde del meato Se inyecta lentamente el material seleccionado hasta que el abultamiento progresivo, habón, oblitere casi por completo el orificio ureteral, en el que puede verse levantado el borde superior del meato en forma de arco (boca enfadada) o quedar el meato encima del habón (forma de volcán). Por norma hay que evitar realizar punciones múltiples, ya que favorecen el extravasado del material. La técnica se realiza ambulatoriamente, completándose aproximadamente en 15 minutos. No hay cantidad específica de material para inyectarse, variando desde $0^{\prime} 2$ a $1 \mathrm{ml}$, juzgándose la ideal por la morfología del habón creado El procedimiento se puede realizar con vejiga llena o vacía, siendo nuestra preferencia a repleción media. Antes de retirar la aguja conviene esperar de 1 a 2 minutos para que se compacte el material, ya que si no puede salir por el sitio de la pun-

TABLA I. DISTRIBUCIÓN DE LOS URÉTERES REFLUYENTES SEGÚN PATOLOGÍA.

\begin{tabular}{|l|c|}
\hline \multicolumn{1}{|c|}{ TIPOS DE REFLUJO V-U } & $\begin{array}{c}\text { NÚMERO } \\
\text { DE } \\
\text { URÉTERES }\end{array}$ \\
\hline PRIMARIO & 457 \\
\hline ASOCIADO A SISTEMA DUPLEX & 76 \\
\hline ASOCIADO A VEJIGA NEURÓGENA & 24 \\
\hline INTERVENIDOS CON CIRUGÍA & 12 \\
ABIERTA & \\
\hline
\end{tabular}


TABLA II. DISTRIBUCIÓN DEL GRADO DE RVU SEGÚN PATOLOGÍA.

\begin{tabular}{|c|c|c|c|c|c|c|}
\hline GRADO & Simple & Dúplex & $\begin{array}{c}\text { Vejiga } \\
\text { neurógena }\end{array}$ & $\begin{array}{c}\text { Cirugía } \\
\text { abierta }\end{array}$ & Total & $\%$ medio \\
\hline II & $109(24 \%)$ & $19(25 \%)$ & $3(13 \%)$ & & 131 & 23 \\
\hline III & $193(42 \%)$ & $30(39 \%)$ & $7(27 \%)$ & & 230 & 40 \\
\hline IV & $119(26 \%)$ & $23(30 \%)$ & $12(50 \%)$ & $9(67 \%)$ & 163 & 29 \\
\hline V & $36(8 \%)$ & $4(6 \%)$ & $2(9 \%)$ & $3(23 \%)$ & 45 & 8 \\
\hline Total & 457 & 76 & 24 & 12 & 569 & 100 \\
\hline
\end{tabular}

ción. Aconsejamos vaciar la vejiga antes de finalizar para evitar molestias.

El paciente permanece en observación de "Hospital de corta estancia" unas 2 a 3 horas antes de dar el alta.

El material empleado ha variado dependiendo de la época en la que se realizó el procedimiento: politetrafluoroetileno desde los años 1988 a 1996, polidimetilsilixano desde 1997 a 1999 y desde 2000 hasta 2006 dextranómero de ácido hialurónico. La razón del cambio ha sido la disponibilidad de material, que en un principio fue único, presentación más adecuada del producto (jeringas unidosis estériles y mejoras en la pistola de inyección) y pasar de los materiales no reabsorbibles a los reabsorbibles.

Para el seguimiento efectuamos una ecocistografía a los tres meses y al año del procedimiento para comprobar la resolución del RVU y los resultados los hemos definido siguiendo dos criterios:

- Curación: ausencia de reflujo en la primera ecocistografía de control.
- Mejoría: Disminución del grado de reflujo que permite un seguimiento médico (grado I y grado II).

Si el RVU se ha corregido en el primer control suspendemos la profilaxis y practicamos el segundo control al año del procedimiento, que sirve para detectar recurrencias, y si sigue resuelto valoramos alta. Si el reflujo no está curado o no puede pasar a vigilancia médica propondremos nueva inyección pasados 3 meses más Se efectúan un máximo de tres intentos endoscópicos para corregir el RVU y si persiste y está indicada su corrección se lleva a cabo una cirugía antirreflujo mediante técnica abierta.

\section{RESULTADOS}

La distribución de los grados de reflujo tratados muestra una preponderancia de los grados III y IV (Tabla II).

Utilizamos tres tipos de materiales, predominado el politetrafluoroetileno con 257 uréteres, siguiéndole el dextranómero de ácido hialurónico con 159 y por último el polidimetilsilixano con 153 (Tabla III).

TABLA III. DISTRIBUCIÓN DEL MATERIAL EMPLEADO.

\begin{tabular}{|l|c|c|c|c|c|}
\hline \multicolumn{1}{|c|}{ MATERIAL } & Simple & Dúplex & $\begin{array}{c}\text { Vejiga } \\
\text { neurógena }\end{array}$ & Quirúrgicos & Total \\
\hline Politetrafluoroetileno & 196 & 46 & 10 & 5 & 257 \\
\hline Polidimetilsilixano & 116 & 25 & 8 & 4 & 153 \\
\hline Dextranómero de ácido hialurónico & 145 & 5 & 6 & 3 & 159 \\
\hline Total & 457 & 76 & 24 & 12 & 569 \\
\hline
\end{tabular}


No se han encontrado diferencias estadísticamente significativas entre los grupos tratados con politetrafluoroetileno, polidimetilsilixano o dextranómero de ácido hialurónico, por lo que analizamos todos los uréteres conjuntamente por grupos de patología y no por material empleado.

En los "RVU simples" la tasa de curación global fue del $88 \%$ (381uréteres), con mejora importante del grado de reflujo en el $7 \%$ (51 uréteres), precisando una reimplantación tipo Cohen en el 5,5\% de los casos (25 pacientes). El 67\% (308 uréteres) obtuvieron la curación con una única intervención (el $87 \%$ en el grado II, $74 \%$ de los grado III, el $55 \%$ de los grado IV y el $70 \%$ de los grado V). Observamos un menor índice de éxitos y mayor necesidad de más inyecciones en los reflujos grado IV y $\mathrm{V}$ (Tabla IV).

En los "RVU asociados a duplicidad" los resultados empeoran, con menos éxitos y mayor necesidad de procedimientos para su resolución. Curamos 59 uréteres $(77 \%)$ sobre 76 uréteres tratados, 13 uréteres (19\%) mejoraron y $4 \%$ precisaron de reimplantación tipo Cohen. La tasa de curación con una única inyección fue del 52\% (40 uréteres) (Tabla V).

En cuanto a los "RVU asociados a vejiga neurógena" se consiguió curación en 20 uréteres (83\%). Con un solo procedimiento 16 uréteres $(67 \%)$ y en $4(16 \%)$ fueron necesarios dos procedimientos. Hubo mejoría significativa del reflujo en 2 uréteres (8\%). Fracasamos en dos (8\%), necesitando de reimplantación tipo Cohen para evitar el deterioro progresivo del riñón (Tabla VI). En algunas ocasiones hubo dificultad para encontrar los meatos debido a la presencia de trabeculaciones marcadas, presentes en algunas de estas vejigas, convirtiendo el procedimiento en más largo y tedioso. No se presentó ninguna otra complicación reseñable.

En los "RVU de uréteres reimplantados mediante cirugía abierta" curamos 12 uréteres (100\%) sobre 12 tratados. Hubo 8 uréteres simples, solucionando el reflujo en
6 con un único procedimiento, mientras que uno precisó de dos procedimientos. En 4 uréteres había duplicidad ureteral asociada, resolviéndose $1(11 \%)$ con un procedimiento y $3(22 \%)$ con 3 inyecciones.

Globalmente se curaron 8 (67\%) de las 12 unidades tratadas con un único procedimiento, mientras que 4 unidades $(33 \%)$ necesitaron 2 procedimientos.

El porcentaje de uréteres curados según el grado de reflujo se puede apreciar en la Tabla VII.

Durante las intervenciones no encontramos complicaciones apreciables, salvo una mayor dificultad técnica secundaria a la anatomía alterada secundaria a la reimplantación previa.

En total el número de uréteres curados ha sido de $496(87 \%)$ y 51 (9\%) han mejorado. Se han intervenido 22 uréteres $(4 \%)$.

Con 1 inyección hemos curado un 68\%, con 2 inyecciones un $16,5 \%$ y con 3 el $1 \%$.

Las complicaciones acaecidas en estos 569 procedimientos, fueron $5(0,8 \%)$ : 1 caso de cistitis hemorrágica que cedió espontáneamente en dos días y 4 pielonefritis que se trataron con antibiótico adecuado según antibiograma. No hemos tenido casos de infecciones urinarias bajas de repetición.

Durante el seguimiento hemos visto recidiva del reflujo previamente curado en 43 uréteres dos de ellos asociado a vejiga neurógena. La mayoría de los casos el reflujo fue grado II respondiendo satisfactoriamente a un nuevo procedimiento endoscópico.

El tiempo de seguimiento ha variado de 1'5 años a 15 años sin evidenciarse ningún tipo de reacción adversa en los pacientes por el tipo de material empleado, ni ninguna clínica sospechosa de migración de partículas de material.

TABLA IV. RESULTADO DE TRATAMIENTO ENDOSCÓPICO DEL RVU SIMPLE.

\begin{tabular}{|c|c|c|c|c|c|c|c|}
\hline Grado & $\mathbf{1}$ & $\mathbf{2}$ & $\mathbf{3}$ & Mejoría & $\begin{array}{c}\text { Cirugía } \\
\text { abierta }\end{array}$ & $\begin{array}{c}\text { \% } \\
\text { Curación }\end{array}$ & Total \\
\hline Inyección & Inyecciones & Inyecciones & & - & 95 & 109 \\
\hline III & $145(75 \%)$ & $19(10 \%)$ & $4(2 \%)$ & $17(9 \%)$ & $8(4 \%)$ & 85 & 193 \\
\hline IV & $65(55 \%)$ & $28(23 \%)$ & $5(4 \%)$ & $13(11 \%)$ & $8(7 \%)$ & 78 & 119 \\
\hline V & $26(72 \%)$ & $9(25 \%)$ & - & $1(3 \%)$ & - & 97 & 36 \\
\hline Total & 332 & 64 & 9 & 36 & 16 & 87 & 457 \\
\hline
\end{tabular}




\section{DISCUSIÓN}

Desde que en 1984 apareció la publicación de O’Donell y Puri (10) sobre el tratamiento endoscópico del $\mathrm{RVU}$ en niños con éxito, el procedimiento se ha ido expandiendo lentamente hasta generalizarse debido a su sencillez, fácil reproducción y buenos resultados. Durante estos años la técnica endoscópica no ha variado y las principales mejoras en el campo del tratamiento endoscópico del RVU han sido en el desarrollo de nuevos materiales. Se buscan materiales que puedan ser introducidos endoscópicamente, que conserven su volumen con el tiempo, que sean biocompatibles, no antigénicos, no carcinogénicos y no migren. Actualmente hay múltiples sustancias pero ninguna ideal $(3,13,14)$. Se pueden clasificar en no reabsorbibles y reabsorbibles.

Dentro de las no reabsorbibles se encuentran la pasta de politetrafluoroetileno y el polidemitilsiloxano y entres las sustancias reabsorbibles destacan el colágeno y el dextranómero de ácido hialurónico.

La pasta de politetrafluoroetileno fue el primer material en utilizarse. Se han logrado buenos resultados en el tratamiento del RVU, con una tasa de éxito superiores al $92 \%$. Está descrito que con la pasta de politetrafluoroetileno puede aparecer una reacción granulomatosa, que puede persistir y aumentar de tamaño durante varios años (15). Se ha sugerido que este fenómeno podría dificultar la intervención quirúrgica abierta, hecho falso según hemos comprobado en nuestra propia serie. A pesar de su eficacia clínica, una gran desventaja es su propensión a la migración (13).

Existen publicaciones contradictorias sobre la posibilidad de migración de la pasta de politetrafluoroetileno. Las partículas de teflón tienen entre 4 y 40 micras, pudiendo pasar a la circulación venosa y ser transportadas por la sangre hasta quedar atrapadas en órganos terminales distantes. (16) Estudios experimentales efectuados por Miyakita han demostrado la falta de migración tras la inyección de una dosis mínima de teflón subureteralmente y tampoco la migración al cerebro tras inyección intravenosa de teflón $(17,18)$. Hay diversas series publicadas en las que los resultados obtenidos son excelentes, con tasas de curaciones mayores del $85 \%$ y escasa morbi-mortalidad. Además en estudios con seguimiento a largo plazo indican que el efecto terapéutico se mantiene sin complicaciones importantes (19-23), hecho confirmado en nuestra serie.

El polidemitilsiloxano se compone de partículas de polidemetilsiliozano vulcanizado, suspendidas en un hidrogel de polivinilpirrolidona. Ha sido el sustituto del teflón debido al mayor tamaño de sus partículas que dificulta la migración, su presentación en unidosis con mejor esterilidad y una pistola de inyección más segura. Se le ha achacado reacciones inmunitarias y posible riesgo de malignización. También se han comunicado casos de migración (13) Nosotros lo hemos utilizamos a partir de $1997 \mathrm{sin}$ apreciar ninguna alteración y con gran efectividad. Se han publicado cifras de curación entre 90 y $91 \%$ (24-27).

El colágeno es un solución con un $95 \%$ de colágeno tipo I y un $5 \%$ de colágeno tipo III, obtenidos del córion bovino. Después de inyectarse, se neovasculariza y estimula la invasión activa de fibroblastos del huésped hasta que, en último término, el implante es sustituido por colágenos de tipos I y III de origen endógeno. No hay migración de partículas y la respuesta inflamatoria frente al implante es leve, sin verse formación de granulomas. Frey en 1995 informa una tasa de éxito del $78 \%(28)$. Presenta varios inconvenientes: Incapacidad de mantener su volumen en el tiempo por su biodegradación junto con la retracción del material inicialmente inyectado, lo que provoca altas recurrencias y altas tasas de retratamiento. También se han descrito reacciones inmunitarias adversas, riesgo de hipersensibilidad aguda y desarrollo de enfermedades del tejido conectivo como lupus eritematoso sistémico, polimiositis y dermatomiositis (13). No se han comunicado migración de partículas ni formación de granulomas.

El copolimero de ácido hialurónico / dextranómero lo desarrollaron Stenberg y Läckgren y es el material empleado en la actualidad. No es inmunogénico, por carecer

TABLA V. RESULTADOS EN DUPLICIDAD URETERAL.

\begin{tabular}{|c|c|c|c|c|c|c|}
\hline Grado & $\begin{array}{c}\mathbf{1} \\
\text { inyección }\end{array}$ & $\begin{array}{c}\mathbf{2} \\
\text { inyecciones }\end{array}$ & Mejoría & $\begin{array}{c}\text { Cirugía } \\
\text { abierta }\end{array}$ & $\begin{array}{c}\% \\
\text { curación }\end{array}$ & Total \\
\hline II & $12(63 \%)$ & $2(11 \%)$ & $5(26 \%)$ & - & 74 & 19 \\
\hline III & $12(38 \%)$ & $10(34 \%)$ & $4(14 \%)$ & $4(14 \%)$ & 72 & 30 \\
\hline IV & $13(56 \%)$ & $6(26 \%)$ & $4(18 \%)$ & - & 79 & 23 \\
\hline V & $3(75 \%)$ & $1(25 \%)$ & - & - & 100 & 4 \\
\hline Total & 40 & 19 & 13 & 4 & & 76 \\
\hline
\end{tabular}


TABLA VI. RESULTADOS EN VEJIGA NEURÓGENA.

\begin{tabular}{|c|c|c|c|c|c|c|}
\hline Grado & $\begin{array}{c}\mathbf{2} \\
\text { Inyección }\end{array}$ & Inyecciones & Mejoría & $\begin{array}{c}\text { Cirugía } \\
\text { abierta }\end{array}$ & $\begin{array}{c}\text { \% } \\
\text { Curación }\end{array}$ & Total \\
\hline II & $3(100 \%)$ & - & - & - & 100 & 3 \\
\hline III & $7(100 \%)$ & - & - & - & 100 & 7 \\
\hline IV & $5(42 \%)$ & $4(34 \%)$ & $1(8 \%)$ & $2(16 \%)$ & 74 & 12 \\
\hline V & $1(50 \%)$ & - & $1(50 \%)$ & - & & 2 \\
\hline Total & 16 & 4 & 2 & 2 & 87 & 24 \\
\hline
\end{tabular}

de moléculas de dextrano libres circulantes, y no migra debido a su forma esférica y al mayor tamaño de las partículas (80 a $120 \mu \mathrm{m})$. Aunque con el tiempo es degradado por hidrólisis, las microesferas de dextranómero producen un efecto de relleno al reclutar fibroblastos y otras células que producen activamente colágeno, formando una masa persistente de tejido blando fibroso (15). Un primer estudio en animales de experimentación constató que un $77 \%$ conservaba su volumen hasta un año después. Posteriormente en estudios con niños se comprobó eficacia con una inyección del 68\%. Ningún paciente presentó reacciones adversas. Queda por demostrar su eficacia a largo plazo, dado el carácter biodegradable del material (13).

Existen también materiales autólogos como los condorcitos que están en fase clínica de su aplicación, con buenos resultados $(13,14)$.

La efectividad varía entre $80-86 \%$ siendo menor en los asociados a duplicidades con un $63 \%$ (29) aunque se debe más a la patología que al tipo de material. A pesar de la diversidad de lo expuesto no parece haber una gran diferencia a la hora de la efectividad entre los distintos materiales. Los resultados de la serie más importante publicada hasta la fecha, el estudio multicéntrico coordinado por Puri y Granata (30), con 12251 unidades renales estudiadas, indican una curación del $75^{\prime} 3 \%$ tras una única primera inyección, un $12 \%$ tras 2 inyecciones, y un $2 \%$ precisaron 3 ó más procedimientos, con una tasa de fallo que indicó una reimplantación ureteral del 4'5\%. Las complicaciones no son frecuentes, apareciendo en nuestra serie en el $0^{\prime} 87 \%$. Dentro de las agudas las más corrientes son: la hematuria que suele ceder espontáneamente, la pielonefritis que precisa de antibióticos y la obstrucción, aunque rara, que se presenta entre un $0^{\prime} 32-0^{\prime} 33 \%$ precisando a veces de derivación y/o intervención $(25,46,1$ mio). Se han referido a largo plazo infecciones urinarias bajas de repetición sin aparición del RVU entre 3'6\%-8\% $(31,32)$.

Los fallos que han precisado de cirugía varían de $1^{\prime} 8-7 \%(30,31,32)$. Las causas son diversas, entre ellas destacan la mala técnica por falta de experiencia, el des-

TABLA VII. CURACIÓN SEGÚN EL GRADO DE RVU.

\begin{tabular}{|c|c|c|c|}
\hline Grado & $\begin{array}{c}\text { Número de uréteres } \\
\text { refluyectes }\end{array}$ & $\begin{array}{c}\text { Número de uréteres } \\
\text { curados }\end{array}$ & Porcentaje \\
\hline II & 131 & 122 & $93 \%$ \\
\hline III & 230 & 200 & $87 \%$ \\
\hline IV & 163 & 132 & $81 \%$ \\
\hline V & 45 & 42 & $93 \%$ \\
\hline Total & 569 & 496 & $87 \%$ \\
\hline
\end{tabular}


plazamiento del habón del material, la perdida de volumen y la extrusión del material $(31,33)$. En nuestro estudio sólo un $4 \%$ de uréteres tratados precisaron tratamiento quirúrgico con cirugía abierta, no encontrando dificultad añadida a la hora de la disección ureteral, ni peores resultados con la reimplantación.

Observamos peores resultados en los casos en que el reflujo se asocia a duplicidad ureteral, precisando de mayor número de inyecciones con menor tasa de curaciones que los casos con reflujo simple, aunque el porcentaje de pacientes que precisan reimplantación ureteral, tras intentar un tratamiento endoscópico, es muy similar a la serie con reflujo simple.

En los casos de RVU con vejiga neurógena, a pesar de una mayor dificultad técnica, se ha referido una curación del $82 \%(34)$, siendo del $87 \%$ en nuestra serie. La recurrencia se suele producir en los primeros meses postratamiento, debido a que en ese periodo de tiempo se producen los procesos de reabsorción de los materiales biodegradables y de los excipientes, la fibrosis reactiva y la movilización del material, procesos que influyen en la conformación final del uréter terminal y del implante. Nosotros realizamos por ello, como otros autores (2), un segundo control a los 12 meses del procedimiento, ya que hemos observado recurrencia del RVU a pesar de un primer control negativo.

Dados los buenos resultados obtenidos, la escasa morbi-mortalidad asociada y la posibilidad de repetir el procedimiento endoscópico si fuera necesario actualmente consideramos en nuestro servicio el tratamiento endoscópico como el tratamiento quirúrgico de elección en los pacientes con RVU, independientemente del grado y del tipo (35).

Parece que tanto el polidemitilsiloxano como el dextranómero de ácido hialurónico son materiales buenos y seguros y no tienen el peligro de migración a distancia que tiene el politetrafluoroetileno.

El material que utilizamos en este momento en nuestro centro es el dextranómero de ácido hialurónico ya que, en nuestra opinión, tiene dos grandes ventajas: por un lado al ser biodegradable minimiza las posibles consecuencias provocadas por una teórica migración, y por el otro su facilidad de manejo, dadas sus características de fluidez y viscosidad.

Actualmente el tratamiento endoscópico constituye una opción primaria para los casos de RVU en que este indicada la cirugía, quedando la vía abierta como segunda opción terapéutica.

\section{BIBLIOGRAFÍA y LECTURAS RECOMENDADAS ("lectura de interés $\mathrm{y}^{* *}$ lectura fundamental)}

1. WALTER, R.D.; DUCKETT, J.W.; BARTOLONE. y cols.: "Secreening school children for urologic disease". Birth. Defect., 13: 399, 1977.
2. CAPOZZA, N.; LAIS, A.; NAPPO, S. y cols.: "The role of endoscopic treatment of vesicoureteral reflux: A 17 year experience". J. Urol., 172: 1626, 2004.

3. PURI, P.: "Endoscopic treatment of vesicoureteral reflux". Pediatric Urology, Gearhart J.P., Rink R.C., Mouriquand W.B. Saunders Company. Philadelphia, 26: 411, 2001.

4. KRAMER, S.A.: "Vesicoureteral reflux". Clinical Pediatric Urology Belman A.B, King L.R, Kramer S.A. Edit: Martin Dunita. London, 25: 749, 2002.

5. BIRMIGHAN REFLUX STUDY GROUP: "Prospective trial of operative versus nonoperative treatment of severe vesicoureteric reflux in children: Five year's observations". BMJ, 295: 237, 1987.

6. Mc LOIRE, G.A.; Mc KENNA, P.H.; JUMPER, B.M. y cols.: "High grade vesicoureteric reflux: Analysis of observational therapy”. J. Urol., 144: 537, 1990.

7. TAMMINEN- MOBIUS, T.; BURNIER, E.; EBEL, K.D. y cols.: "Cessation of vesicoureteral reflux for 5 years in infants and children allocated to medical treatment". J. Urol., 148: 1662, 1992.

8. REPORT ON THE MANAGEMENT OF PRIMARY VESICOURETERAL REFLUX IN CHIDREN. Baltimore. American Association, pp., 1-88, 1997.

9. MATOUSCHEK, E.: "Sobre un Nuevo concepto para el tratamiento del reflujo vesicoureteral". Arch. Esp. Urol., 34: 385, 1981.

10. O’DONNELL, B.; PURI, P.: "Treatment of vesico-ureteric reflux by endoscopic injection of Teflon". Brit. Med. J., 289: 7, 1984.

11. SCHULMAN, C.C.: "Traitement endoscopique du reflux vesico-ureteral chez l'enfant". Chir. Pédiatr., 27: $181,1986$.

12. ANGULO, J.M.; ARTEAGA, R.; RODRÍGUEZ ALARCÓN, J. y cols.: "Nuestra experiencia en el tratamiento endoscópico con teflón del reflujo vésicoureteral en niños". Cir. Pediatr., 8: 161, 1995.

13. KERSHEN, R.; ATALA, A.: "Nuevos tratamientos inyectables para la incontinencia y el reflujo vésico-ureteral. Clínicas de Urología de Norteamérica. Urología reconstructiva". McGraw-Hill Interamericana, 1: 87, 1999.

14. ATALA. A.; PETERS, S.C.; RETIK, A.B. y cols.: "Endoscopic treatment of vesicoureteral reflux with a selfdetachable ballon system". J. Urol., 148: 724, 1992.

15. PURI, P.; PIRKER, M.; MOHANAM, N. y cols.: "Subureteral Dextranomer/ Hyaluronic acid injection as first line treatment in the management of high grade vesicoureteral reflux". J. Urol., 176: 1856, 2006.

16. BORGATTI, I.R.; TETTAMANTI, A.; PICCINELLI, P.: "Lesión cerebral en un niño sano después de una inyección periureteral de teflón”. Pediatrics (ed. Esp.), 42: 121,1996

17. MIYAKITA, H.; PURI, P.: "Particles found in lung and brain following subureteral injection of polytetrafluoroethylene paste are not Teflon particles". J. Urol., 152: 636, 1994.

18. MIYAKITA, H.; O’BRIAN, D.S.; PURI, P.: "Ausence of brain parenchymal damage following intravascular injection of polytetrafluoroenthylene paste". Euro. Urol., 34: 233, 1998.

19. BHATTI, H.A.; KHATTAK, H.; BOSTON, V.E.: "Efficacy and causes of failure of endoscopic subereteric in- 
jection of teflon in the treatment of primary vesicoureteric reflux". Br. J. Urol., 71: 221, 1993.

20. CAPOZZA, N.; PATRIOLO, M.; LAIS, A. y cols.: "Endoscopic Treatment of vesicoureteral reflux: Twelve years experience". Urol. Int., 67: 228, 2001.

21. JOYNER, B.D.; ATALA, A.: "Endoscopic substances for the treatment of vesicoureteral reflux". Urology, 50: 489, 1997.

22. MONTERO, M.; MÉNDEZ, R.; TELLADO, M. y cols.: "Estudio comparativo del tratamiento del reflujo vesico-ureteral en la edad pediátrica: Revisión de una serie de 636 unidades refluyentes". Cir. Pediatr., 12: 144, 1999.

23. PELÁEZ MATA, D.; ALVAREZ ZAPICO, J.A.: "Estado actual del tratamiento del reflujo vesicoureteral. Análisis de nuestra experiencia”. Cir. Pediatr., 14: 112, 2001.

24. HERZ, D.; HAFEZ, A.; BAGLID, D. y cols.: "Efficacy of endoscopic subureteralpolydimethylsiloxane injection for treatment of vesicoureteral reflux in children: A north American Clinical report". J. Urol., 166: 1880, 2001.

25. AL-HUNAYAN, A.A.; KEHINDE, E.O.; ELSALAM, M.A. y cols.: "Outcome of endoscopio treatmente for vesicoureteral reflux in children using polydimethylsiloxane". J. Urol., 168: 2181, 2002.

26. ABOUTALEB, H.; BOLDUC, S.; UPADHYA, Y. y cols.: "Subureteral polydimethylsiloxane injection versus extravesical reimplantation for primary low grade vesicoureteral reflux in children: A comparative estudy". J. Urol., 169: 313, 2003.

27. ELDERS, J.S.: "Editorial comment". J. Urol., 167: 1810, 2002.
28. FREY, P.; JENNY, P.; HRZOG, G.B.: "Endoscopic suburetric collagen injection (SCIN): A new alternative treatment of vesicoureteric reflux in children". Experience in 82 refluxing ureters". Ped. Surg. Int., 6: 287, 1991.

29. LACKGREN, G.; WAHLIN, N.; SKOLDENBERG, E. y cols.: "Endoscopic treatment of vesicoureteral reflux with dextranomer / hyaluronic acid copolymer is effective in either double ureters or a small kidney". J. Urol., 170: 1551, 2003.

30. PURI, P.; GRANATA, C.: "Multicentrer survey of endoscopic treatment of vesicoureteral reflux using polytetrafluoroethylene". J. Urol., 160: 1007, 1998.

31. CHERTIN, B.; COLHOUN, E.; VELAYUDHAM, M. y cols.: "Endoscopio treatment of vesicoureteral reflux: 11 a 17 years of folloup". J. Urol., 167: 1443, 2002.

32. LACKGREN, G.; WAHLIN, N.; SKOLDENBERG, E. y cols.: "Longterm followup of children treated with dextranomer/hyaluronic acid copolymer for vesicoureteral reflux". J. Urol., 166: 1887, 2001.

33. DIAMOND, D.A.; CALDAMONE, A.A.; BAUER, S.B. y cols.: "Mechanisms of failure of endoscopic treatmente of vesicoureteral reflux based of endoscopic anatomy". J. Urol., 170: 1556, 2003.

34. MISRA, D.; POTTS, S.R.; BROWN, S. y cols.: "Endoscopic treatment of vesicoureteric reflux in neurologenis bladder -8 years experience". J. Pediatric. Surg., 31: 1262, 1996.

35. DAWRANT, M.; MOHANAN, N.; PURI, P.: "Endoscopic treatment for high grade vesicoureteral reflux in infants”. J. Urol., 176: 1847, 2006. 